\title{
REFERENCE MODEL BASED DESIGN OF TOOL LANDSCAPES FOR RAIL INFRASTRUCTURE ENGINEERING
}

\author{
Holm, T.; HoRn, S.; LEHMANN, O. \& SEIDEL, H.
}

Abstract: The rail automation domain will, in the near future, face drastic changes. For instance, the use of a standardized electronic representation of signaling planning will become an integral part of the communication of customer requirements. These changes pose challenges for the manufacturers of rail automation systems, who must adapt their engineering tools accordingly. In order to do so, these challenges have been analyzed as part of this paper. The analysis has been used to derive a reference model for the integrated, tool-based engineering of rail automation systems. Further it influences the working process between the different engineering groups and enables potentials e.g. for a fully digital and seamless engineering process. It is applied today at Siemens AG in order to evaluate engineering tools for rail automation as well as to design entire engineering tool landscapes from scratch.

Key words: engineering, reference, model, rail, automation, engineering
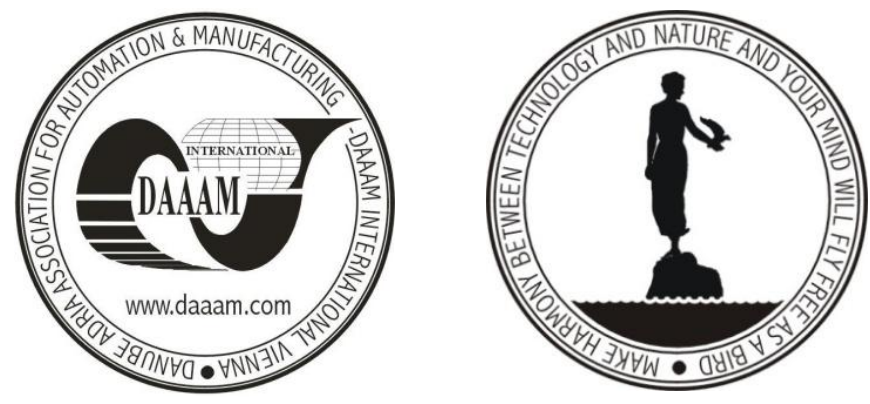

Authors' data: Dr.-Ing. Holm, T[imo]*; Dr.-Ing. Horn, S[tefan]*; Dr.-Ing. Lehmann, O[thmar]**; M.Sc. Seidel, $\mathrm{H}[$ eiko]**, *Siemens AG, Werner-von-Siemens-Str. 61, 91052 Erlangen, Germany, **Siemens AG, Ackerstr. 22, $38126 \quad$ Braunschweig, Germany, timo.holm@siemens.com, stefan.horn@siemens.com, othmar.lehmann@siemens.com, heiko.seidel.ext@siemens.com

This publication has to be referred as: Holm, T[imo]; Horn, S[tefan]; Lehmann, O[thmar] \& Seidel, H[eiko] (2012). Reference Model Based Design of Tool Landscapes for Rail Infrastructure Engineering, Chapter 23 in DAAAM International Scientific Book 2012, pp. 267-276, Published by DAAAM International, ISBN 9783-901509-86-5, ISSN 1726-9687, Vienna, Austria

DOI: $10.2507 /$ daaam.scibook.2012.23 
Holm, T.; Horn, S.; Lehmann, O. \& Seidel, H.: Reference Model Based Design of ...

\section{Introduction}

Business with rail automation plants in general and electronic interlockings in particular faces drastic changes. Driven mainly by market forces, the electronic representation of customer requirements - more accurately the electronic representation of signaling planning - is introduced as an integral part and interface in communications between the customer and plant manufacturer. The procedural changes needed within the engineering process to harvest the potential of this particular trend as well as other trend are not fully understood yet.

This paper gives an overview of these challenges that manufacturers of rail automation solutions must face and aims to help evaluate and design engineering tools and entire landscapes by introducing a reference model of an integrated engineering tool landscape for rail interlocking systems.

An integrated engineering tool landscape further enables new approaches in order to establish concepts regarding concurrent engineering, e.g. seamless and efficient interoperation between different engineering groups. Focused on collaboration a strategy for the implementation of an IT-supported workflow is investigated. For this purpose a holistic workflow management is necessary to obtain a high-grade process quality and a greater degree of automation within the engineering process. Thus the contribution further focuses on challenges and concepts to increase the cooperation between different users within engineering workflow.

\section{Trends in rail automation engineering and engineering levers}

The simultaneous occurrence of three major trends is unique for the rail automation domain:

- $\quad$ Electronic representation of signaling planning - This trend is driven mainly by market forces. Standardized modeling languages to represent parts of the customer requirements are introduced as an integral part and as a means of communication between the customer and plant manufacturer. This trend features considerable potential for increasing productivity, since it not only has a major media brake but also enables all stakeholders to push tool support for consistency checking and overall automation efforts. One prominent example for standardized data formats in this context is RailML (Nash et al., 2010), which is begin currently extended in order to cover interlocking applications.

- Mechatronic modeling and integrated engineering data management - The concept of mechatronic objects as discipline-spanning entities representing reusable units in the context of plant modeling is often paired with the integrated and lifecycle phase spanning management of engineering data, see also (Hundt, 2012). This trend has several origins, for instance the fields of model based engineering, mechatronic modeling and holistic plant data management. In order to support the collaboration of all disciplines involved, additional functionalities are needed. For instance concepts such as users, roles and rights management as 
well as methods to represent engineering processes and workflows (see figure $1)$.

- Test automation - An interlocking system as a result of rail automation engineering activities usually needs to comply with the highest safety integrity levels (SIL 4) of IEC EN 61508 (IEC EN 61508, 2010). Therefore a lot of effort is put into testing individual engineering artifacts as well as their interaction. In order to optimize this cost driver the automation of major parts of these activities is evaluated.

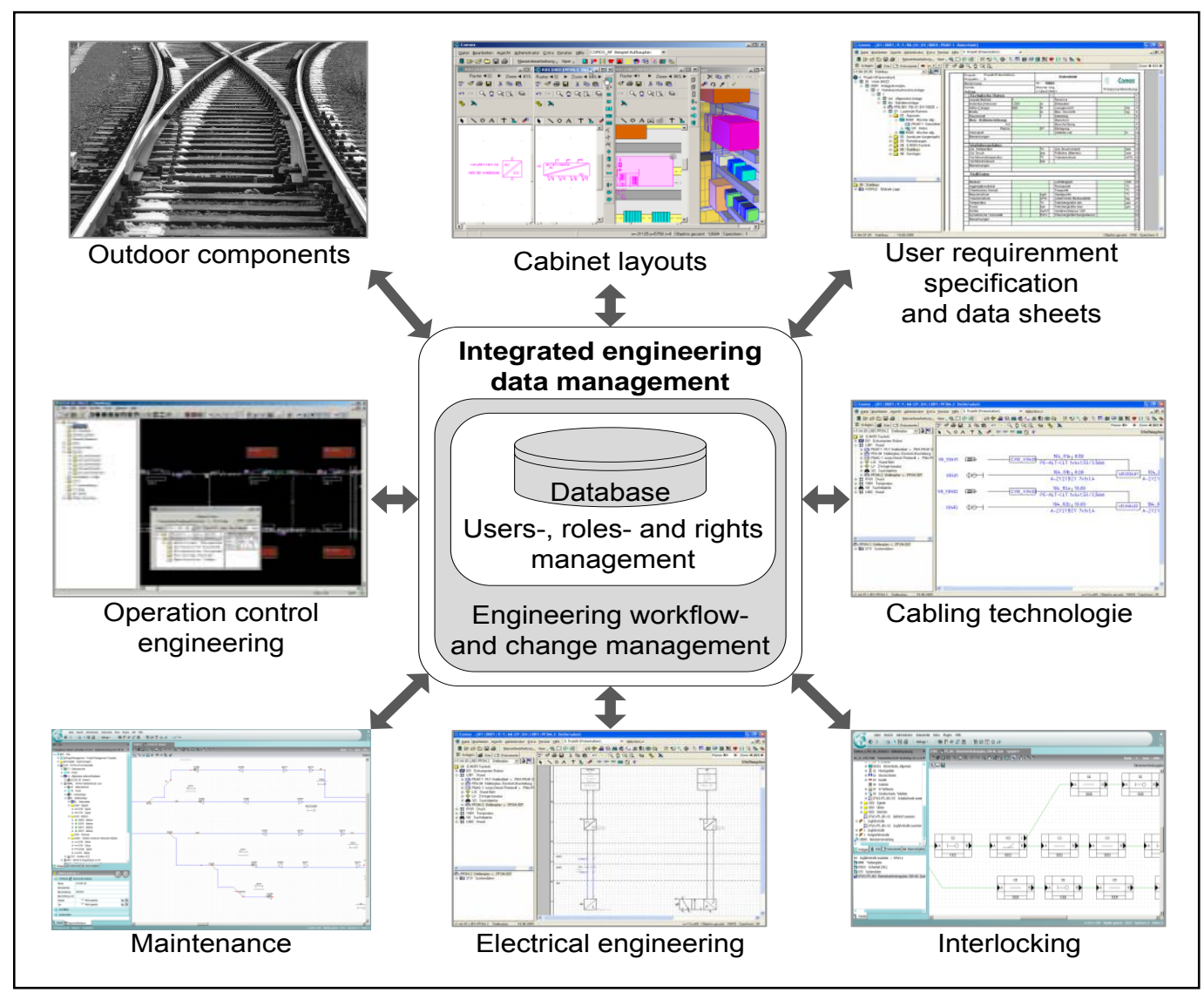

Fig. 1. Integrated engineering data management

However, the levers to harvest efficiency potentials in engineering are well known and described accordingly in system engineering theory (Loewen et al., 2005): - Integration of disciplines - usually different disciplines are involved in designing a plant. Examples are electrical engineering and interlocking control programming. Activities associated with these disciplines are executed during the same plant lifecycle phase by a multitude of engineers in parallel. The integration of these disciplines aims to support an engineer e.g. for the identification and resolution of changes within the engineering data. Thus parallel engineering activities demand an efficient workflow management, as crucial concept for concurrent engineering. Consistent data warehouse improves the engineering quality as well as cooperation between these actors.

- $\quad$ Plant lifecycle integration - the manufacturing process phases of a plant may not be conceived as independent of one another. Instead, those plant lifecycle 
phases need to be designed in a continuous manner in order to leverage the possible benefit potentials. The artifacts (outputs) of one phase for instance should ideally be fitted to support the processes of the following phase.

- $\quad$ Reuse - the results of an engineering process, usually referred to as engineering artifacts, are often of a recurring pattern. In order to save time and effort, the reuse of those artifacts - within one and the same project but also in a projectspanning manner - is an important benefit lever.

- Coverage - artifacts which are to be reused within a project context ideally cover as much as possible: They span multiple disciplines and are, - generally speaking - big, which means they cover as many requirements and functions as possible while simultaneously being flexible and of universal applicability.

The interrelations between the four levers mentioned are depicted in figure 2 .

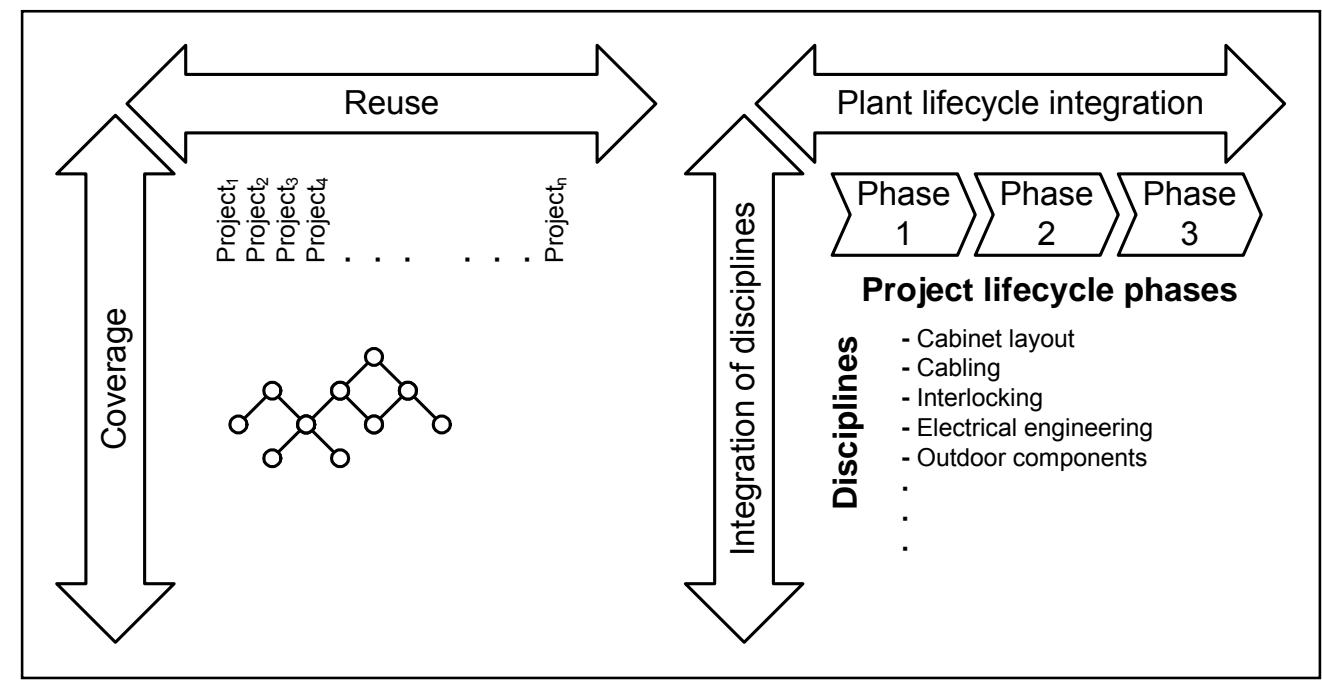

Fig. 2. Four levers in engineering (Loewen et al., 2005)

\section{Reference modeling}

In order to be able to sufficiently consider the above-mentioned trends when designing an engineering tool landscape, knowing those levers is not enough. Additional information regarding their relevance and place of action is needed. In order to gather this information, a reference model-based approach has been used. The result of these design activities, which have been based on the methodology described by Probst in (Probst, 2003), is a generic engineering tool landscape for the rail infrastructure automation domain. It is supposed to serve as common practice and is currently being adopted by Siemens Rail Automation.

Figure 3 depicts the mentioned architecture, which consists of three functional component-groups that can be assigned to three corresponding layers:

- Electronic representation of Signaling planning - In future, an external

planner - usually mandated by the customer - will provide a customer file which contains a standardized, electronic representation of parts of the customer requirements. Since many these standards, country-specific as well as customerspecific, will have to be supported, conversion into a homogenous, uniform 
representation is necessary. This representation enables the supplier to automate parts of the plan checking process using rules to support the user.

- Integrated engineering data management - Assuming the plan checking process has been completed successfully, the electronic representation of signaling planning is transferred into the data model sub-component of engineering data management. Ideally it predetermines at least parts of the finished models used by the component Generator in order to produce engineering artifacts such as BOMs, plans, PLC code or user documentation. This data model must be accessed in a controlled manner. Therefore components for the representation of users, their roles within the engineering process and permissions for (parts of) the data model must exist. However changes made to the engineering data model must not corrupt or leave it in an invalid state. Therefore a set of rules running inside a rule engine is triggered whenever changes are made. Changes are made either by the engineers using authoring components, so-called external, pre-existing or internal editors or by a automation runtime environment. Since modification patterns to the data model are highly standardizable, this component offers a way of relieving engineers from this type of activity, e.g. mass-renaming or mass-duplicating of elements. These activities as well as users, roles and rights and also the scheme, which defines the underlying structure of the data model, have to be managed by an administrator. An additional component targets the reuse-lever, see also figure 2 . (Loewen, 2005). The product repository contains information of predefined submodels - so called products - from which the final, project specific engineering data model can be built. Usually product managers are put into place to manage these reusable artifacts.

- Test environment - When the engineering activities of an interlocking project come to an end, the above mentioned engineering artifacts need to be inspected, tested and approved by a certified technical inspection authority in order to meet the regulatory requirements of local government agencies or bodies. To support the corresponding processes three components are necessary. A test case generator automatically derives tuples of stimuli and expected results and forms test cases, which are gathered in catalogs and passed on to a component called "test manager". The test case generator uses information taken from the electronic representation of signaling planning, which has not yet been modified in any way as a result of the engineering process. This is necessary since results of the engineering process itself are to be tested. This requires a separate approach for the generation of a reference standard. 


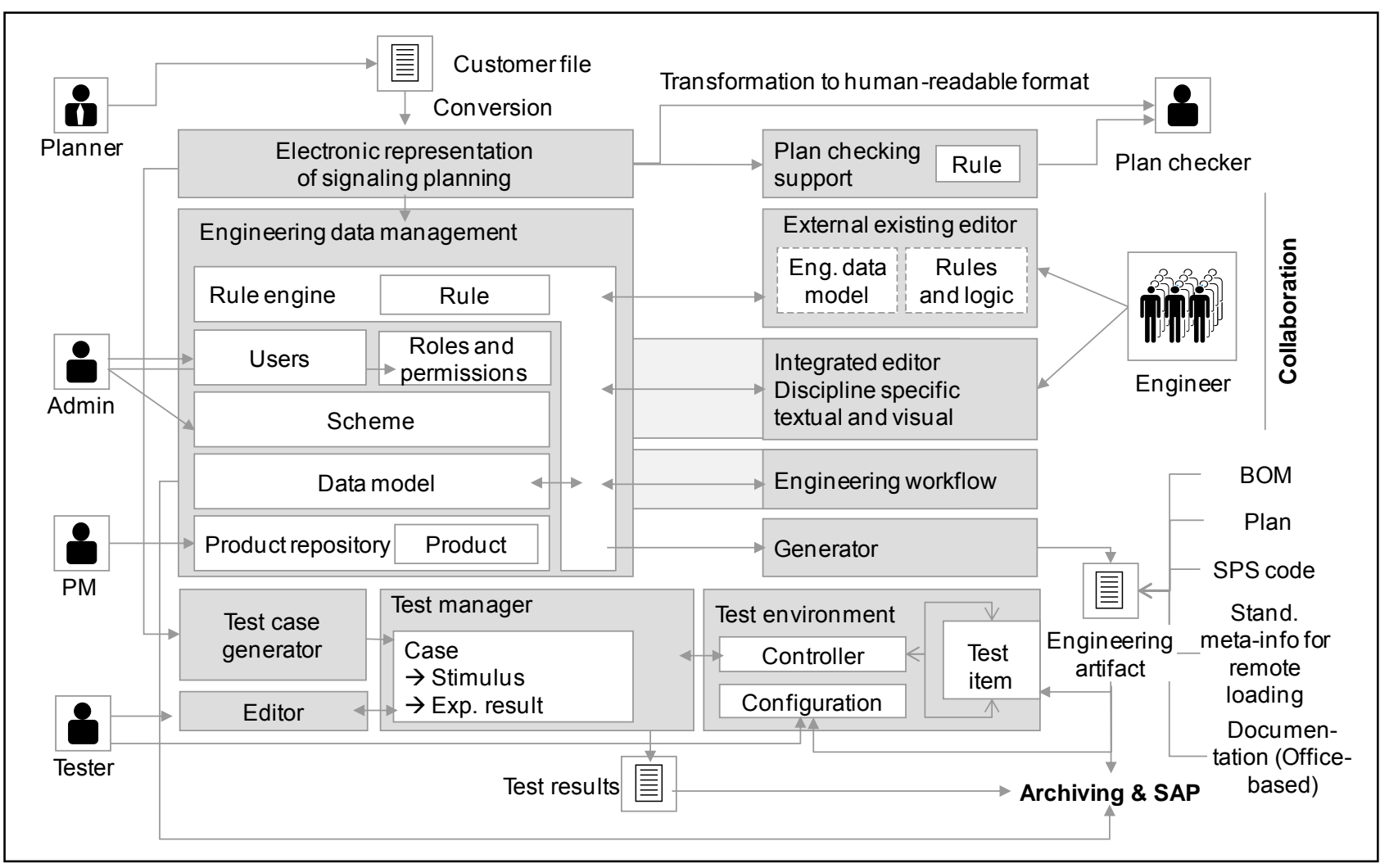

Fig. 3. Reference model for engineering tools in rail automation

\section{Concurrent engineering support by means of workflows}

Efficient collaboration within engineering projects is a major challenge. The integration of a holistic workflow management is essential for gearing engineering groups within projects. Potentials and goals of an efficient workflow management are the reduction of the project duration, increasing engineering quality i.e. to minimize mistakes, avoidance of rework during commissioning and ensure transparency of the engineering progress. IT-supported cooperative workflow management further creates bridges between heterogeneous Computer Aided Engineering (CAE) tools as well as their data models respectively. The complexity of overall engineering process and tool interaction can be handled better by modeling engineering activities explicitly. Modeling is the most important engineering technique. Models help us to understand and analyze large and complex problems. This notion of abstraction is a challenge by creating IT-based workflows, what is confirmed by (Kramer, 2007). Further thereby offer the possibility to increase the level of automation e.g. by data generators or merging mechanisms. Due to the transparency of the engineering progress and consistent data maintenance is it possible to acting of changes in other engineering groups.

The benefit of an integrated workflow management in the domain of rail automation results by the improvement of consistency of the engineering data between the engineering groups. For example, if the functional structure - the topology of the rail system - is changed, the electrical planning and the relevant component of the interlocking system can be adjusted by a synchronization activity immediately. 


\subsection{Workflow implementation}

For transferring the activities within workflows it is essential to define workflow patterns for each activities. According to the concept for the definition of mechatronic objects, how described in (Böhm, 2012) a high degree of abstraction of those workflow pattern enable a common use for different engineering disciplines over the whole lifecycle. The following action items are identified as basic for the development engineering workflows (Balzert, 2009):

- abstraction of engineering activities

- modularization of workflows for the specific engineering groups

- $\quad$ structuring the activities of engineering disciplines specific workflows

- $\quad$ hierarchization or determine the sequence of activities

- $\quad$ binding and coupling of workflow elements in dependency of the engineering object relations

- information hiding to manage the complexity

- localization of information and referenced data and objects

- verbalization to create transparency about the activities

For the implementation of a holistic workflow management, these principles have to be applied. It is necessary to abstract the engineering activities to identify and create engineering patterns. These global patterns are used as a top-level workflow. The workflow patterns have to be refined to create sub-models for the different engineering groups and connected to build hierarchical and structured workflows. To achieve a high flexibility for the configuration of an engineering workflow dependencies between activities must be analyzed and transformed into a formal and digital representation in order to allow a computerized interpretation of these workflows. As illustrated in figure 5, the modularization of the workflow elements capsulate activities and a flat hierarchy between the engineering disciplines allow dynamical execution and those to avoid a stagnation of particular engineering groups.

Binding and coupling of the workflow elements with other engineering disciplines-specific workflows must be defined for a consistent and transparent engineering state. The encapsulation of necessary information is useful to provide only target group specific data. Irrelevant information leads to confusion of the user and must be hidden. For complex engineering solution is it necessary to handle only relevant and precise data. Further a central data management as proposed in chapter 3 avoids complex searching and synchronization algorithms over distributed and often encapsulated data sources. Thus the aforementioned binding and coupling of the engineering data is a basic requirement for this concept to provide all relevant data for a certain engineering activity. Furthermore it is advisable to describe the engineering workflows verbally to ensure that it is understood across involved engineering disciplines and to enable transparency according to the workflow progrss. These described points have dependencies among each other. Figure 5 describes the dependencies of these principles. 


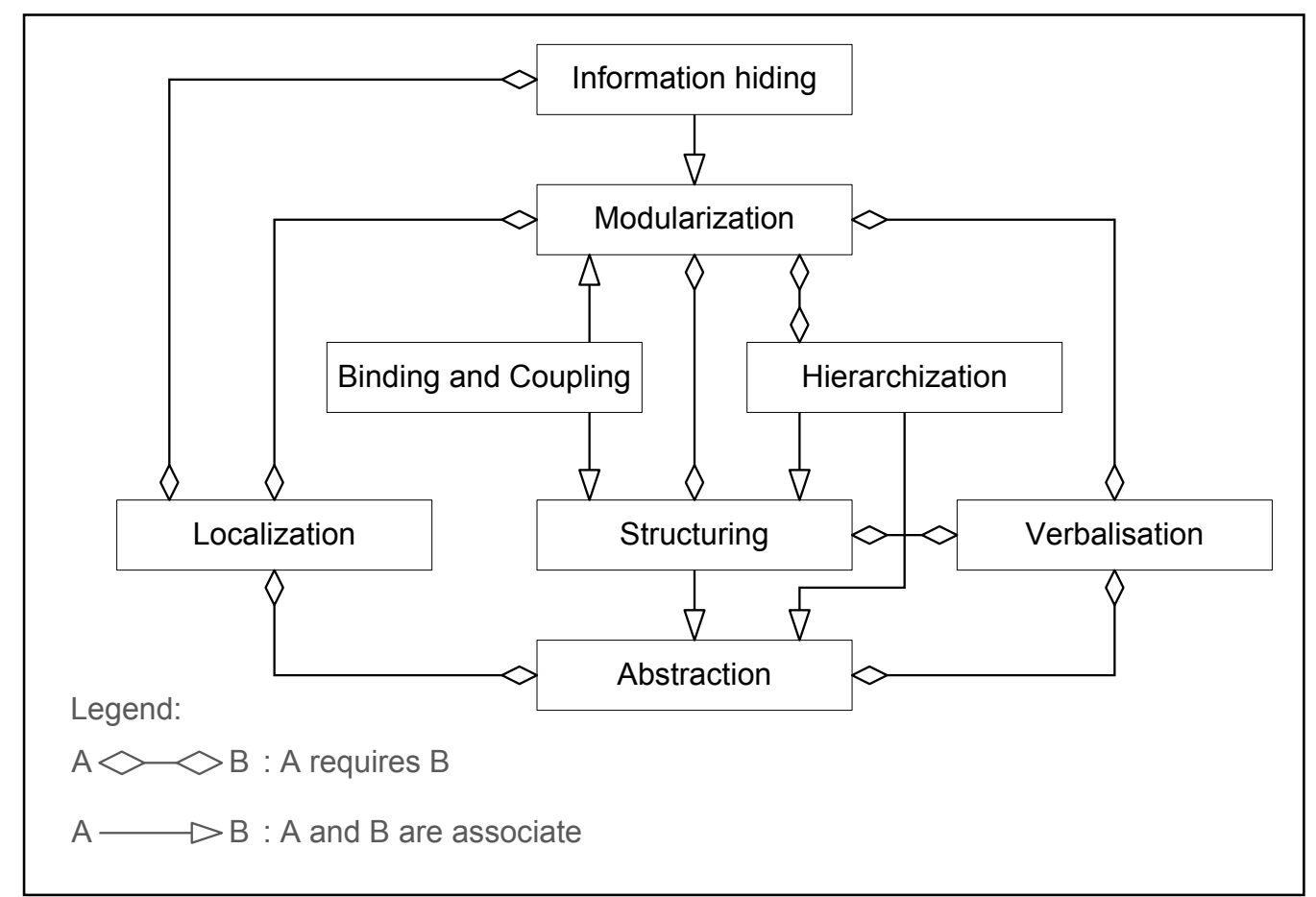

Fig. 4. Dependencies of derivation workflow principles

\subsection{Workflow automation}

The main focus of future implementations with engineering tools is to increase the automation of engineering activities. Without workflow management the control functions are only applied to encapsulated parts without knowing the whole process, e.g. the function "create signal" instead of the complete workflow "processing outdoor facilities". IT-supported workflow management should support the process holistically, so that the user is able to focus the particular task and will be lead through the process.

The architecture of CAE tools is often established over several years. Thus it is often difficult to redesign a complete tool chain. IT-supported workflow management can also work like a middleware, which integrates these existing engineering tool structures to synchronize collaborative engineering data and the engineering progress between the engineering disciplines. The challenge for the abstraction of workflow patterns is to identify and formalize the "start and die end" of those activities, i.e. trigger events must occur to start as well as to finished an automated workflow process. Furthermore it has to be investigated, which steps of a workflow have to be finished as prediction of a workflow pattern. For this requirement the principle of binding and coupling is important to detect relations between workflow activities and engineering objects respectively. Further common and IT-based synchronization mechanisms, e.g. trigger events as shown in figure 5, is a mandatory requirement to guarantee data consistency in case of parallel engineering processes. This synchronization mechanism should be embedded in the workflow management concept. The necessary data to finish an activity are determined by a suitable commit mechanism.

A possible solution to keep the engineering data consistent and to finish an engineering activity recommended in (Sokolov, 2012). Synchronously activities are 
executed by a request-reply pattern, where the result occurs immediately. There is possible to check the data before every discipline-crossing engineering step. Parallel or asynchronous activities have mostly a request-wait-continue pattern if the result for continue will occur far in the future. In parallel collaborative engineering procedures it is not possible to create sequential points of handover. Rather a perpetual online synchronization- and merging flow must be occurring by means of workflows.

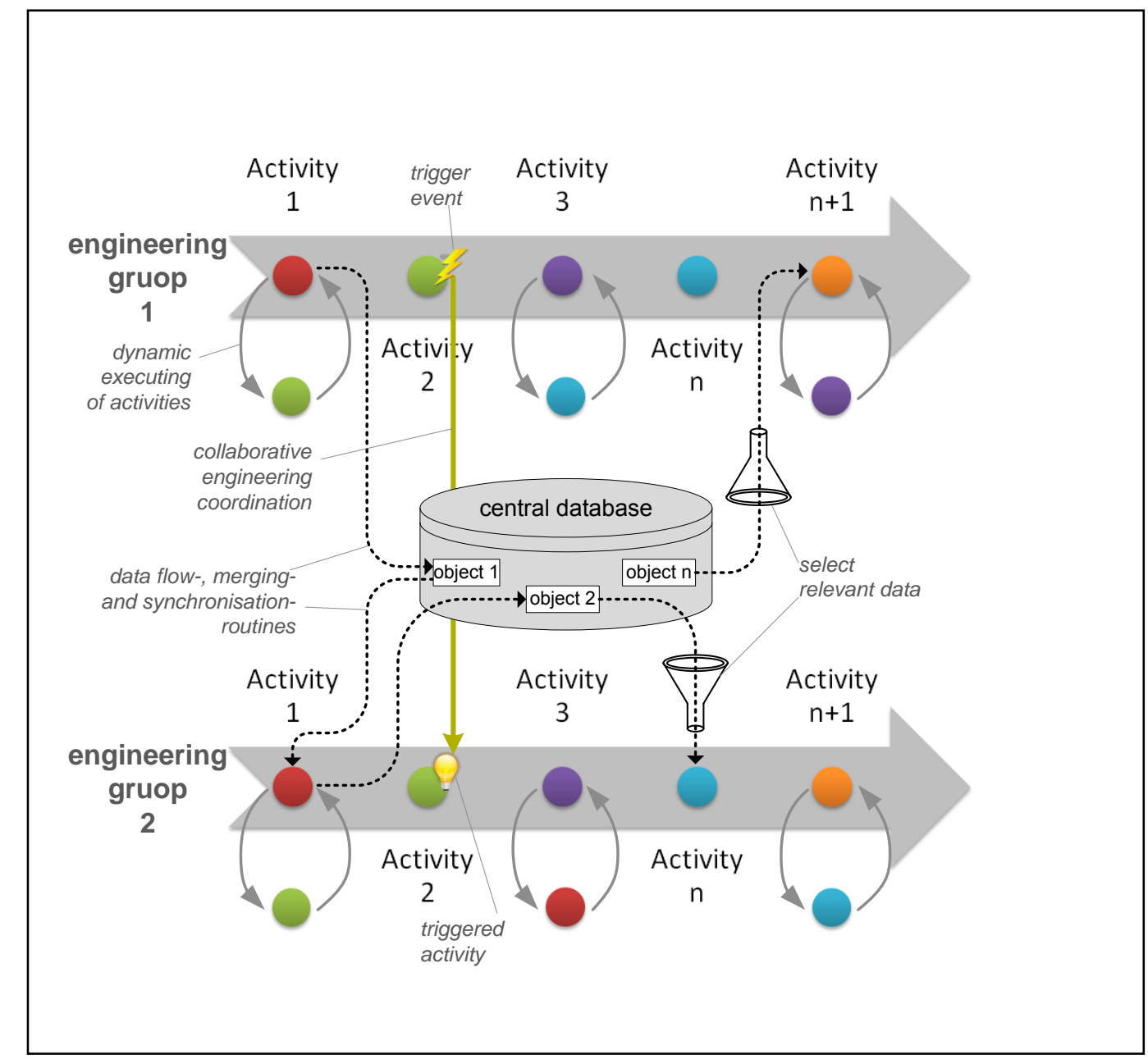

Fig. 5. Dynamic workflow and IT-based add-ons

\section{Conclusion and outlook}

In order to design an engineering tool landscape for rail automation systems that considers above mentioned trends, all reference components have to be covered by one or more current information systems. To decide which design alternatives might be better or worse, additional evaluations have to take place (Holm et al., 2008). The reference architecture is essential to determine which evaluation criteria (e.g. software quality) are relevant for which component.

To handle the increasing complexity of engineering tasks, the implementation of IT-based workflow management is strongly recommended. The trend is to implement integrated mechanisms within CAE tools to support workflow management. Already existing approaches focus on the ERP-level mostly or focus on an abstract level and 
must be detailed for the application within technical systems. For the definition of common standards of technical engineering activities further discussion has be to continued to identify, which generic engineering workflow elements already exist and how the level of automation regarding developed engineering processes can be increased further.

\section{References}

Balzert, H. 2009: Base concepts and requirements engineering. 3rd edition. Heidelberg : Spektrum Akademischer Verlag, 2009

Böhm, B.; Gewald, N.; Köhlein, A., et al. 2012: Roadmap for the Development of Mechatronic Objects. In: conference report Automation 2012. Baden-Baden : VDI Wissensforum GmbH, VDI/VDE, 2012

Holm, T.; Luschmann, C.; Delgado, A. and Amberg, M. (2008) A Reference Model Based Approach for the Evaluation of Industrial Plant Service and Asset Management Tools, Annals of DAAAM for 2008 \& Proceedings of the $19^{\text {th }}$ International DAAAM Symposium, Katalinic, B. (Ed.), pp. 601-602, 9-771726967007, Trnava (Slovak Republic), October 2008, DAAAM International, Vienna (Austria)

IEC EN 61508 (2010). Functional safety of electrical/ electronic/ programmable electronic safety-related systems - Part 0: Functional safety, IEC Swiss, 28318-7816-0, Geneva

Hundt, L. (2012): Seamless data exchange for the behavior description of automation systems: A contribution for the data management for the engineering of production sites, $\mathrm{PhD}$, Otto-von-Guericke University Magdeburg, Germany

Durchgängiger Austausch von Daten zur Verhaltensbeschreibung von

Automatisierungssystemen: Ein Beitrag zum Datenmanagement beim Engineering von Produktionsanlagen

Kramer, J. 2007: Is Abstraction the Key to Computing? In: Communications of the ACM, 2007

Löwen, U.; Bertsch, R.; Böhm, B.; Prummer, S. and Tetzner, T. (2005) Systematization of industrial plant engineering, atp Automatisierungstechnische Praxis, Vol. 50, I. 4, pp. 54-61

Nash, A.; Hierlimann, D.; Schuette, J. and Krauss, V.P. (2010). RailML - a standard data interface for railroad applications, In: Timetable Planning \& Information Quality, Hansen, I., (ed.), 3-10, WIT Press, 1-84564-500-6, Ashurst, Southamption

Probst, C. (2003). Referenzmodelle für IT-Service-Informationssysteme, Logos Verlag, 978-3832501617, Berlin

Sokolov, S.; Fichtner, H.-P.; Cihlar, Z., et al. 2012: A process-orients process model for support of knowledge based planning tasks in plant engineering. In: conference report Automation 2012. Baden-Baden : VDI Wissensforum GmbH, VDI/VDE, 2012 